\title{
Protein aggregation profile of the human kinome
}

\author{
Ricardo Graña-Montes ${ }^{1}$, Ricardo Sant'Anna de Oliveira ${ }^{2}$ and Salvador Ventura ${ }^{1}{ }^{*}$ \\ 1 Institut de Biotecnologia i de Biomedicina and Departament de Bioquímica i Biologia Molecular, Universitat Autònoma de Barcelona, Bellaterra (Barcelona), Spain \\ 2 Instituto de Bioquímica Médica, Universidade Federal do Rio de Janeiro, Rio de Janeiro, Brazil
}

Research Topic: From structural to molecular systems biology: experimental and computational approaches to unravel mechanisms of kinase activity regulation in cancer and neurodegeneration

\section{Edited by:}

Matteo Barberis, Humboldt University Berlin, Germany; Max Planck Institute for Molecular Genetics, Berlin, Germany

Reviewed by:

David James, Garvan Institute of Medical Research, Australia

Paolo Tortora, University of

Milano-Bicocca, Italy

*Correspondence:

Salvador Ventura, Institut de Biotecnologia i de Biomedicina, Departament de Bioquímica i

Biologia Molecular, Parc de Recerca UAB, Mòdul B, Universitat

Autònoma de Barcelona, Bellaterra (Barcelona) E-08193, Spain.

e-mail:salvador.ventura@uab.es
Protein aggregation into amyloid fibrils is associated with the onset of an increasing number of human disorders, including Alzheimer's disease, diabetes, and some types of cancer. The ability to form toxic amyloids appears to be a property of most polypeptides. Accordingly, it has been proposed that reducing aggregation and its effect in cell fitness is a driving force in the evolution of proteins sequences. This control of protein solubility should be especially important for regulatory hubs in biological networks, like protein kinases. These enzymes are implicated in practically all processes in normal and abnormal cell physiology, and phosphorylation is one of the most frequent protein modifications used to control protein activity. Here, we use the AGGRESCAN algorithm to study the aggregation propensity of kinase sequences. We compared them with the rest of globular proteins to decipher whether they display differential aggregation properties. In addition, we compared the human kinase complement with the kinomes of other organisms to see if we can identify any evolutionary trend in the aggregational properties of this protein superfamily. Our analysis indicates that kinase domains display significant aggregation propensity, a property that decreases with increasing organism complexity.

Keywords: protein kinases, protein aggregation, amyloid, protein evolution, AGGRESCAN

\section{INTRODUCTION}

Most polypeptides need to fold into specific three-dimensional structures to perform their biological functions (Dobson, 2003). Only the correctly folded forms of these proteins remain soluble in the cell and are able to interact with their molecular targets (Daggett and Fersht, 2009). Therefore, protein misfolding impairs cell fitness and is being found linked to an increasing number of human degenerative diseases. In these disorders, misfolded conformers establish non-native intermolecular contacts that result in their deposition into insoluble amyloid aggregates in the intraor extracellular space (Chiti and Dobson, 2006). All these assemblies display a common cross- $\beta$ motif (Nelson and Eisenberg, 2006). However, the ability to form amyloid-like structures is not restricted to a subset of disease-linked proteins and this conformation may be accessed by most, if not all, proteins in living organisms, from bacteria to human, irrespective of their native fold (Dobson, 2004; Jahn and Radford, 2005; de Groot et al., 2009). In fact, the molecular interactions leading to the formation of amyloids are similar to those promoting the folding and functional assembly of proteins (Linding et al., 2004; Castillo and Ventura, 2009). As a result, folding and aggregation pathways are continuously competing in the cell.

Globular proteins are soluble in their biological environments. However, when their stability is compromised by genetic mutations or environmental conditions, local unfolding might promote the exposure to the solvent of aggregation-prone regions previously protected in the native state (Ventura et al., 2004; Ivanova et al., 2006). To confront this danger, protein sequences have evolved strategies to reduce aggregation propensity (Rousseau et al., 2006b; Monsellier and Chiti, 2007; Tartaglia et al., 2007; Castillo et al., 2011). The selective pressure against protein aggregation is stronger for proteins involved in essential cellular functions (Tartaglia and Caflisch, 2007; Chen and Dokholyan, 2008; de Groot and Ventura, 2010), as it might be the case of kinases. Nevertheless, even those globular proteins selected to be highly soluble cannot avoid the presence of aggregation "sensitive" stretches in their sequences (Sabate et al., 2012). Accordingly, we have recently shown that a cancer associated point mutant of human nucleoside diphosphate kinase A displaying reduced conformational stability forms amyloid fibrils under close to physiological conditions (Georgescauld et al., 2011). Similarly, a partially folded intermediate of phosphoglycerate kinase has been shown to self-assemble into amyloid fibrils (Damaschun et al., 2000; Agocs et al., 2010).

The aggregation propensities of proteins are determined to a large extent by their sequences and the intrinsic properties that govern the aggregation of proteins have been already identified. This has allowed the development of a set of algorithms able to predict aggregation-prone regions in protein sequences as well as the overall aggregation propensity of polypeptides (Castillo et al., 2010; Hamodrakas, 2011). Several of these programs are well suited for the analysis of large protein sets, among them AGGRESCAN, an algorithm previously developed by our group (Conchillo-Sole et al., 2007; de Groot et al., 2012), which displays a high power to predict in vivo protein aggregation (Belli et al., 2011). Different predictive algorithms have been used to analyze the overall aggregation properties of complete proteomes, from bacteria to human (Tartaglia et al., 2005; Rousseau et al., 2006b; Monsellier et al., 2008; de Groot and Ventura, 2010). Here we address the intrinsic aggregational properties of protein 
sequences belonging to the same super-family in different organisms. In this way, we have analyzed the aggregation propensities of the protein kinase complements ("kinomes") of budding yeast, fly, mouse, and humans using AGGRESCAN.

\section{RESULTS}

\section{AGGREGATION PROPERTIES OF KINOMES}

We computed the aggregation properties of the complete kinomes of $S$. cerevisiae (104 proteins, all containing a single kinase domain), D. melanogaster (197 domains in 194 proteins), M. musculus (520 domains in 511 proteins), and H. Sapiens (508 domains belonging to 497 proteins) using AGGRESCAN. The following parameters were calculated (Figure 1 and "Materials and Methods"):

1. The average aggregation propensity of the sequence (Na4vSS).

2. The frequency of occurrence of aggregation-prone regions (APR), i.e., the number of aggregating peaks for each 100 protein residues (NnHS).

3. The average aggregating potency of the detected aggregation peaks (THSAr), i.e., the area of the peaks that lies above the detection threshold, normalized by the protein length.

4. The average aggregating potency of residues above the detection threshold (AATr), independently if they are clustered in

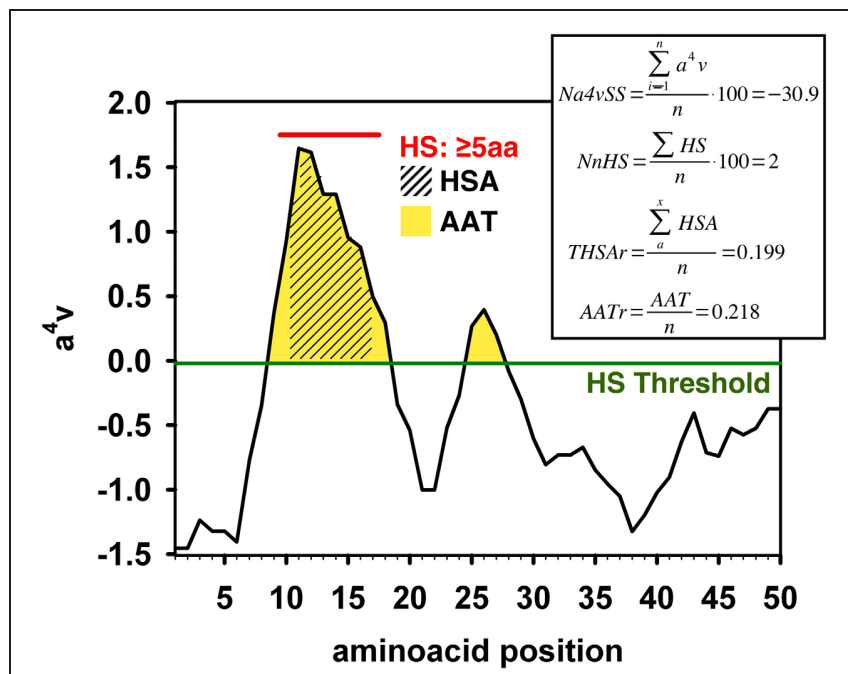

FIGURE 1 | Example of the AGGRESCAN output with the different parameters calculated by this algorithm. The aggregation profile is represented as the value of the experimentally derived parameter $a^{4} v$ (de Groot et al., 2005) plotted against the query sequence. An overall value of this parameter is given for the whole sequence by dividing the a4v sum for all positions over the number of amino acids an multiplying by 100 (Na4vSS). An aggregation-prone region (APR) or Hot-Spot (HS) is detected whenever a stretch of 5 or more consecutive amino acids, none of them being a proline, with a $4 \mathrm{v}$ values above the HS Threshold (HST) is found. The normalized number of Hot Spots ( $\mathrm{NnHS}$ ) is calculated as the number of Hot-Spots detected divided by the sequence length and multiplied by 100 . The area of the profile above the threshold gives an idea of the aggregation potential of the sequence or a certain region; it can be calculated for the whole profile (AAT) or limiting it to the detected Hot-Spots (HSA). The area values are normalized dividing by the number of amino acids in the input sequence. aggregating peaks or not, i.e., the area of the surface above the detection threshold.

We retrieved the sequences corresponding to kinase domains in the full-length proteins for the different kinomes and analyzed their aggregation properties. Surprisingly, the calculated average aggregation propensity Na4vSS was positive in the AGGRESCAN scale in all cases, which suggests a certain intrinsic propensity to aggregate for these domain sequences (Figure 2A). Na4vSS of $1.56,1.42,1.03$, and 0.75 were calculated for yeast, fly, mouse, and human kinomes, respectively. Na4vSS values reflect the average propensity of all the proteins in a dataset. To compare the distribution of domains displaying positive aggregation propensity in the different species, relative to proteins in the Swiss-Prot database, we binned Na4vSS values into 100 groups and calculated the deviation between the human and the rest of kinomes for bins in which Na4vSS $>0$.

$$
d_{(x-\text { human })}=\sum_{i=1}^{N} F\left(N a 4 v S S_{x}\right)-F\left(N a 4 v S S_{\text {human }}\right)
$$
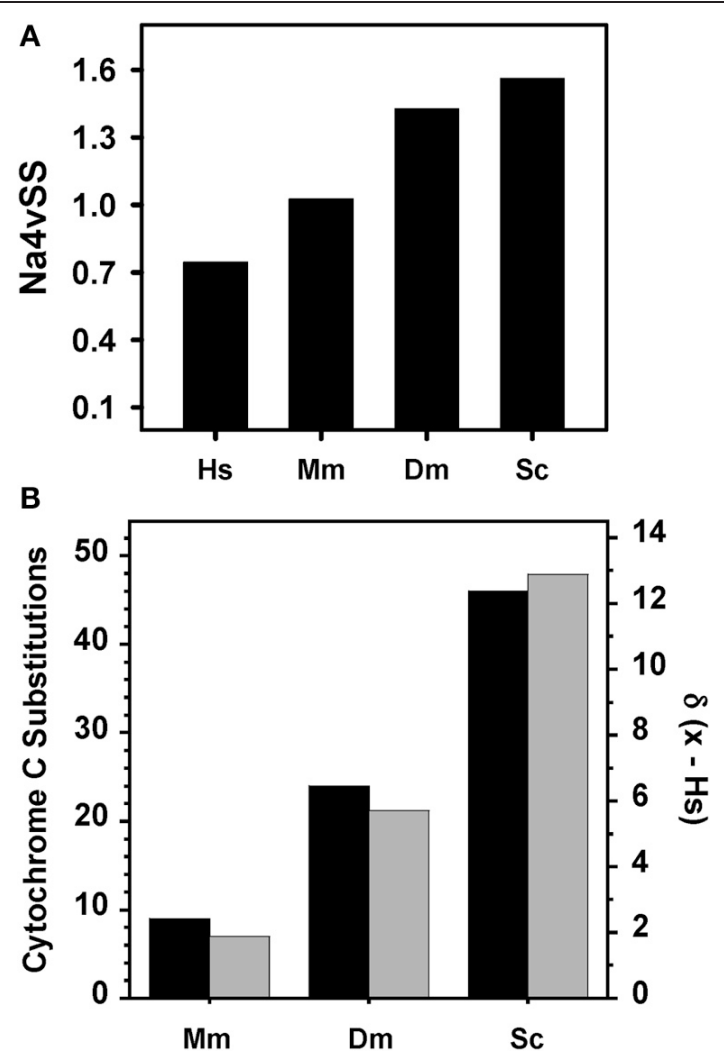

FIGURE 2 | Relationship between organism complexity and aggregation properties of kinase domains. (A) Average aggregation propensity (Na4vSS) for the complete dataset of kinome domains of human $(H s)$, mouse $(M m)$, fruit fly $(D m)$, and budding yeast $(S c)$. (B) Deviation in the distribution of aggregation prone domains ( $\mathrm{Na} 4 \mathrm{vSS}>0$ ) between human and the rest of species (gray, calculated according to Equation 1) compared with the number of amino acid differences in cytochrome $\mathrm{c}$ between human and other species (black). 
where $F\left(\mathrm{Na}_{4} v S_{x}\right)$ corresponds to the frequency of this bin in the organism $x$ and $F\left(N a 4 v S S_{\text {human }}\right)$ is its frequency in the human kinome.

The calculated deviations match well with the evolutive distances in the phylogenetic tree of cytochrome c (Dayhoff et al., 1972) (Figure 2B). Therefore, for kinase domains, it appears that aggregation propensity decreases as we ascend in the evolutionary scale.

We explored the reasons for the different aggregation propensities observed in the kinase domains of different species. The frequency of aggregating peaks NnHS is approximately four in all species (Figure 3A). This value is lower in yeast than in humans and therefore it cannot account for the observed differences in overall aggregation propensity. In contrast, the THSAr values follow the trend observed for Na4vSS, indicating that despite sharing similar number of aggregating peaks, the aggregation potency of these regions decreases with organism complexity (Figure 3B). This became more obvious when we compared the cumulative THSAr frequencies in distant organisms, yeast and human (Figure 3C). The 25\% of the human kinase domains have a low THSAr $(<0.1)$ in contrast to $5 \%$ of yeast domains. On the contrary, 20\% of yeast domains display a high THSAr value $(>0.15)$ while only $10 \%$ of human domains are included in this set. A similar, trend is observed for AATr values, yet another measure of the aggregation propensity of the sequence (Figures 3D,E).
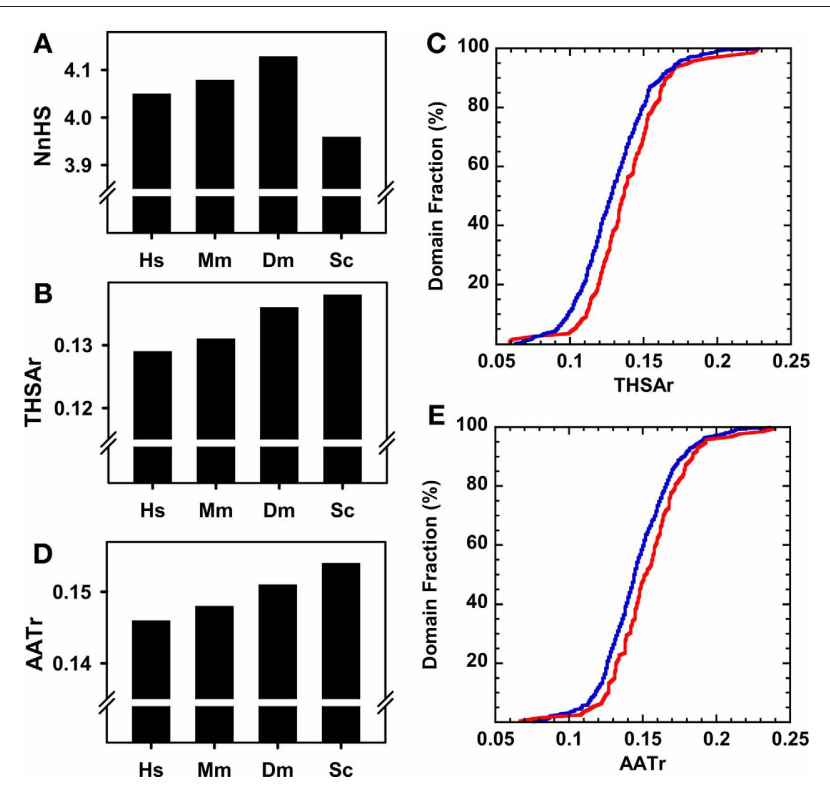

FIGURE 3 | Aggregation properties of complete kinase domain datasets of different organisms. (A) Normalized number of Hot-spots (NnHS). (B) Total Hot-spot area per residue (THSAr). (C) Distribution of the THSAr value along the whole dataset. (D) Area of the aggregation profile above the Hot-Spot Threshold per Residue (AATr). (E) Distribution of the AATr value over the complete kinase domain dataset. In (A), (B), and (D): human $(H s)$, mouse $(\mathrm{Mm})$, fruit fly $(\mathrm{Dm})$, and budding yeast $(\mathrm{Sc})$. In (C) and (E): the blue line corresponds to the human dataset and the red line to the yeast kinome.
To see whether the differences in aggregation propensity of human and yeast sequences might result from an amino acid compositional bias in these species, we compared the amino acid content of yeast and human kinase domains with the average composition of the proteins deposited in Swiss-Prot (Figure 4A). Following the trend described above, both human and yeast kinase domains are, on the average, enriched in residues with high $\beta$-aggregation propensity (C, F, I, L, N, Q, V, and Y) and depleted in residues with low $\beta$-aggregation propensity $(A, G, H$, K, P, and R) (Tartaglia et al., 2005). However, these trends are more evident in yeast domains (Figure 4B), in agreement with their overall higher predicted aggregation properties.

\section{AGGREGATION PROPERTIES OF THE HUMAN KINOME}

We addressed how the aggregation properties of complete human kinase proteins and their domains compare to those of folded proteins. We used the SCOP-derived database ASTRAL40 (Chandonia et al., 2004) and randomly selected 500 sequences in order to obtain a database similar in size to the human kinome (508 domains and 497 proteins). Proteins in the ASTRAL40 database display higher Na4vSS values than the full-length human kinase protein set (Figure 5A). In addition, on the average, the frequency of aggregation-promoting regions is lower in human kinases than in the ASTRAL40 dataset (Figure 5B). Morevover, kinases tend to have less effective aggregating regions than the selected set of human folded proteins (Figures 5C,D). If we only consider the kinase domains in these proteins we observe the opposite trend. The $60 \%$ of kinase domains display positive $\mathrm{Na} 4 \mathrm{vSS}$, in contrast to the $22 \%$ of proteins in the ASTRAL40 dataset. In addition, only $2 \%$ of kinase domains have highpredicted solubility (Na4vSS $<-10$ ), whereas the $30 \%$ of folded human proteins display this property.

When we compare human kinase domains with the correspondent full-length proteins, their aggregation properties appear to be strikingly different. For all the parameters, their cumulative frequencies run parallel but always with higher values for the domains alone (Figures 5A-D). This arises from a higher density of aggregating peaks in the domains, displaying also higher potency, compared to the complete protein in which they reside,

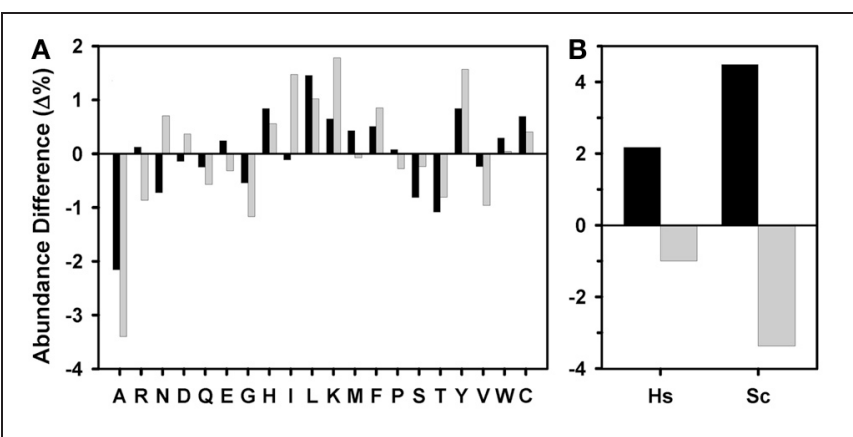

FIGURE 4 | Amino acid composition of kinase domains. (A) Differential amino acid composition of the human (black) and yeast (gray) kinase domains and (B) differential proportion of residues with high (black) and low (gray) $\beta$-aggregation propensity in human ( $\mathrm{Hs}$ ) and yeast $(\mathrm{Sc})$ kinase domains, both relative to the amino acid composition of the Swiss-Prot database ensemble (release 2012_07). 


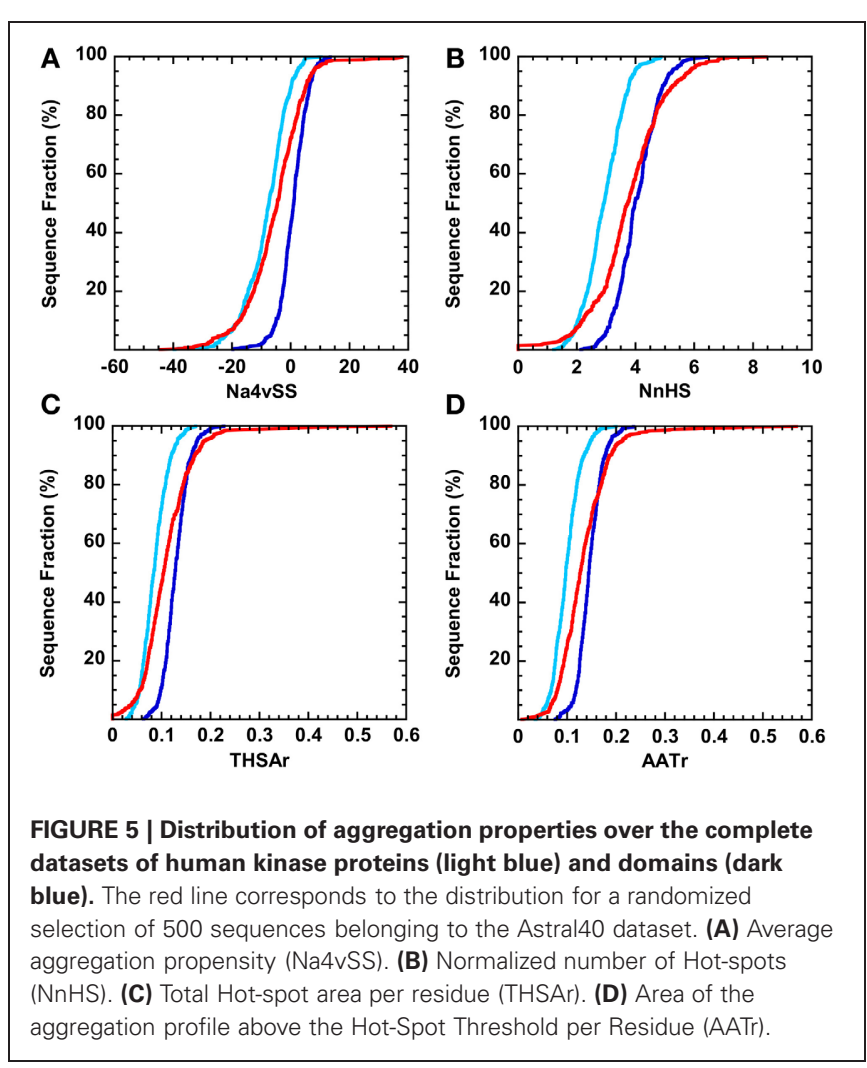

as shown in Figures 6A-C. It has been shown that intrinsically unstructured proteins (IUPs) and segments are inherently less aggregation-prone than globular proteins (de Groot and Ventura, 2010). Therefore, we wondered if there is any relationship between Na4vSS values and the presence of disordered regions in full-length human proteins. With this aim we calculated the intrinsic unfolding propensity of the $10 \%$ protein kinases with the lowest and highest associated Na4vSS values, respectively, using the FoldIndex algorithm. Proteins displaying low intrinsic aggregation propensity are enriched in disordered regions (Figure 6D). This behavior is common to all kinomes, as illustrated in Figure 6D where we compare human and yeast proteins.

\section{AMYLOIDOGENIC REGIONS IN HUMAN KINASE DOMAINS}

AGGRESCAN is a good predictor of intracellular aggregation propensity and of the regions driving this process; however it is not aimed to identify regions that self-assemble into ordered amyloids, albeit aggregation-prone and amyloidogenic regions coincide in many cases (Rousseau et al., 2006a). To extend our analysis, we have also evaluated the presence of patterns corresponding to hexa-peptides that form amyloid-like fibrils as deduced from experimental studies by de la Paz and Serrano (de la Paz and Serrano, 2004). The patterns were detected using ScanProsite (http://prosite.expasy.org/scanprosite/). One thousand and twenty-nine hits were obtained in 435 out of the 508 active domain sequences that include the human kinome dataset, thus indicating that the presence of sequences able to form amyloid structures, if exposed to solvent, is frequent in kinase domains, many of the sequences containing more than one amyloidogenic stretch.
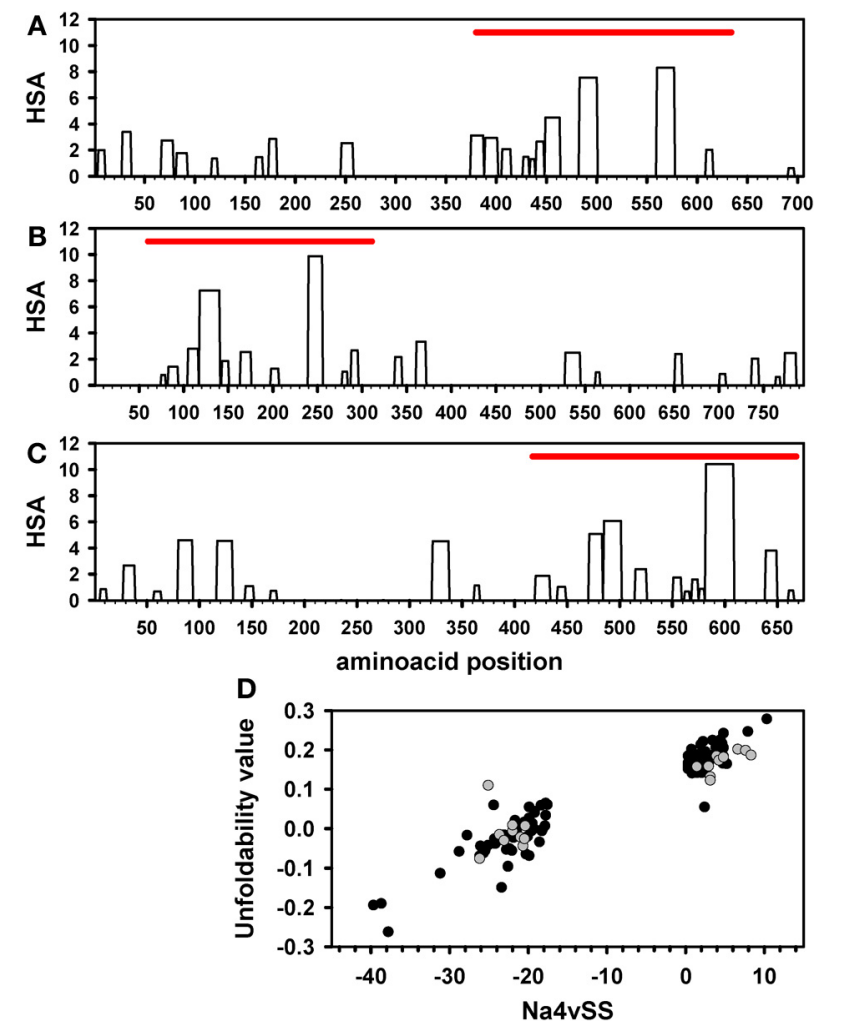

FIGURE 6 | Aggregation prone-regions and intrinsic disorder in kinases. AGGRESCAN Hot-Spot Area (HSA) profile for individual kinase proteins: (A) PKCt (AGC), (B) MARK1 (CAMK), and (C) BMX (TK). The position of the corresponding kinase domain is represented by a red bar. (D) Intrinsical unfolding propensity of the $10 \%$ kinase proteins with the lowest and highest Na4vSS values in the human (black) and yeast (gray) datasets. Intrinsical unfolding propensity predictions were performed using the Foldlndex algorithm.

\section{AGGREGATION AND AMYLOIDOGENIC PROPERTIES OF HUMAN KINASE GROUPS}

Typical protein kinase domains share a common catalytic core consisting of a small, mostly $\beta$-sheet, $\mathrm{N}$-terminal subdomain and a larger, mostly $\alpha$-helical, C-terminal subdomain (Taylor and Radzio-Andzelm, 1994). Despite sharing a common structural core, typical human protein kinases show significant sequential divergence and can be classified in 119 different families corresponding to 9 major groups (Manning et al., 2002b), excluding atypical protein kinases, which do no have structural similarity to the rest of protein kinases. The proteins in a given group are sequentially related. Therefore, we explored if the different groups have characteristic aggregative and amyloidogenic properties. For statistical purposes we analyzed only those groups containing at least 40 sequences: AGC (67 domains), CAMK (69 domains), CMGC (64 domains), Other (80 domains), STE (47 domains), Tyrosine kinase (93 domains), and Tyrosine kinase-like (41 domains). This subset accounts for $89 \%$ of human protein kinase sequences. In Figure 7 we compare the Na4vSS values for the kinase domains and the complete proteins. With the exception of those in the heterogeneous group Other, the 


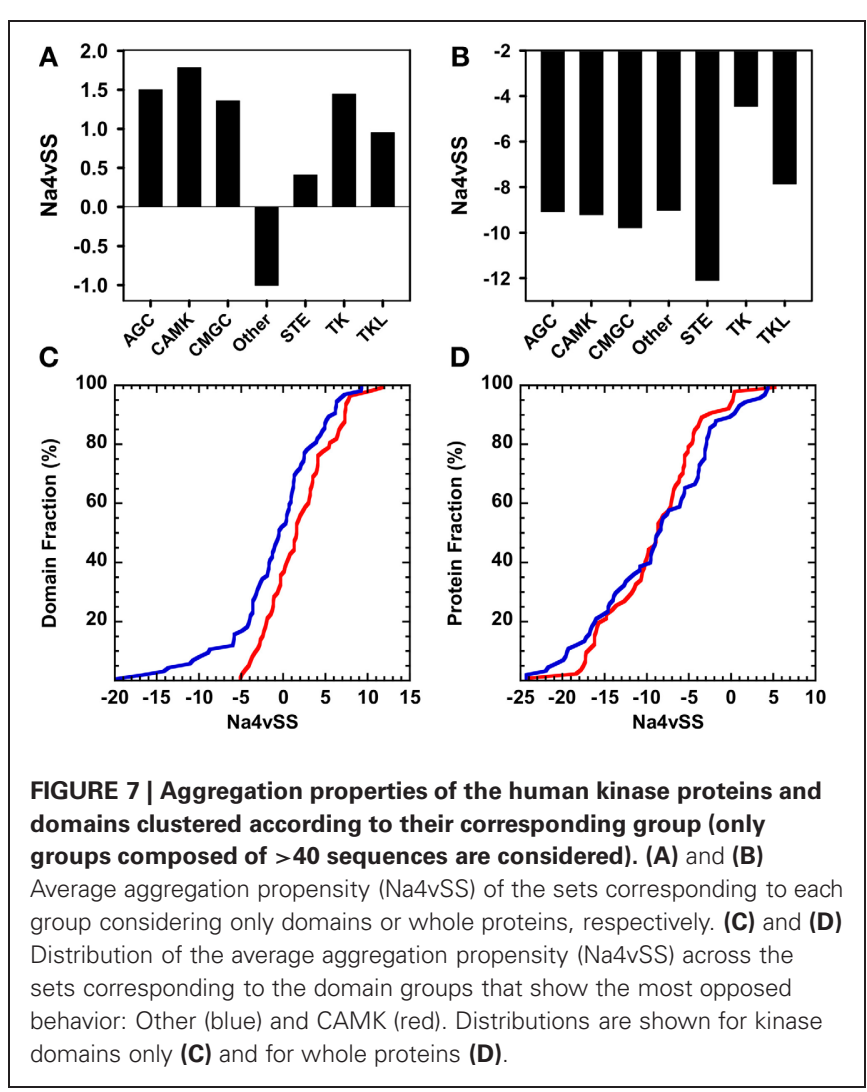

domains in the rest of the groups display positive average aggregation propensity (Figure 7A), with CAMK and STE groups displaying the highest and lowest aggregation propensities, respectively. These differences might reflect functional constrains, since kinases in the CAMK group tend to form complexes by autoassociation whereas STE kinases act transducing signals from the surface of the cell to the nucleus and should be inherently soluble. All full-length proteins display negative Na4vSS values (Figure 7B). The analysis of Na4vSS cumulative frequencies in different groups does not show any correlation between the aggregation propensity of the domain and that of the protein within it is included (Figures 7C,D).

We used the structural alignment reported by Scheeff and Bourne (Scheeff and Bourne, 2005) to map the above mentioned amyloidogenic hexa-peptides over the three-dimensional structures of kinase domains. Thirteen non-redudant structures, representative of the domains in the different human groups, were selected for the analysis (Figure 8). About $90 \%$ of the predicted amyloidogenic regions overlap with secondary structure elements in the kinase domains. These stretches are sequence dependent and, therefore, they map in different regions in the different domains; however the region comprising the well conserved $\beta$-hairpin formed by $\beta$-sheets 4 and 5 at the $\mathrm{N}$-terminal subdomain seems to be specially amyloidogenic. Importantly, the hydrophobic side chains in these two $\beta$-sheets are usually buried inside the structure, thus preventing the aggregation of the native domain under physiological conditions. The amyloidogenic regions in the mitogen-activated protein kinase-activated protein kinase 2, belonging to the CAMK group, which include $\beta$-sheets 4 and 5, are shown in Figure 9 over the domain structure.

\section{AGGREGATION PROPERTIES OF PROTEIN KINASE GROUPS IN DIFFERENT SPECIES}

To test if the observed relationship between domains aggregation propensity and organisms complexity is maintained at the group level, we compared the Na4vSS of human kinase groups with those in yeast, fly, and mouse (Figure 10). It is important to note that the human kinome contains two and five times more kinases than the kinomes of fly and yeast, respectively. Therefore, the number of sequences in the considered groups differs between organisms. A strict correlation between aggregation propensity and organism complexity is seen for domains belonging to kinases in the AGC, CMG, Other, and TK Tyrosine kinase groups. However, deviation from this principle is observed in the CAMK group in which yeast domains appear to be less aggregation-prone than those in multicellular organisms. This might reflect the fact that three out of the seven yeast-specific kinase subfamilies and $30 \%$ of the specific yeast kinase genes belong to the CAMK group (Manning et al., 2002a). They mediate essentially unicellular-specific functions (Ball et al., 2000), including osmotic and other stress responses, which require a significant degree of solubility.

We addressed whether the location of catalytically important residues might constraint the evolution of aggregation propensity. To this aim, we analyzed the presence of conserved catalytic residues inside aggregation-prone stretches in representative proteins corresponding to different groups in human, fly, and yeast (Figure 11). The results indicate that, independently of the protein group and species, a significant amount of catalytic residues are embedded in aggregation-prone regions, likely limiting the evolution of these regions toward more soluble sequences.

\section{DISCUSSION}

Computational predictions of the aggregation propensities of proteomes in different organisms suggest that minimization of protein aggregation may act as an important evolutionary constrain, shaping proteins and cellular machineries (Castillo et al., 2011). This negative selection pressure has been suggested to modulate the aggregation propensity of protein sequences according to their biological context (Rousseau et al., 2006b; Tartaglia et al., 2007; Monsellier et al., 2008; Castillo and Ventura, 2009; Tartaglia and Vendruscolo, 2009; de Groot and Ventura, 2010). In this line, a previous analysis of the overall aggregation tendency of complete proteomes, including those of yeast, fly, mouse, and human, has shown that this value decreases with increasing organism complexity and longevity (Tartaglia et al., 2005). This trend results from the fact that higher organisms contain fewer sequences with high aggregation propensity, but specially because their proteomes contain a higher proportion of IUPs. In addition, the force of natural selection against the aggregation of a given protein depends on the selective contribution of this protein to the organism fitness (Chen and Dokholyan, 2008; Villar-Pique et al., 2012). Therefore, despite the analysis of complete proteomes has contributed significantly to our present understanding on how aggregation modulates protein 


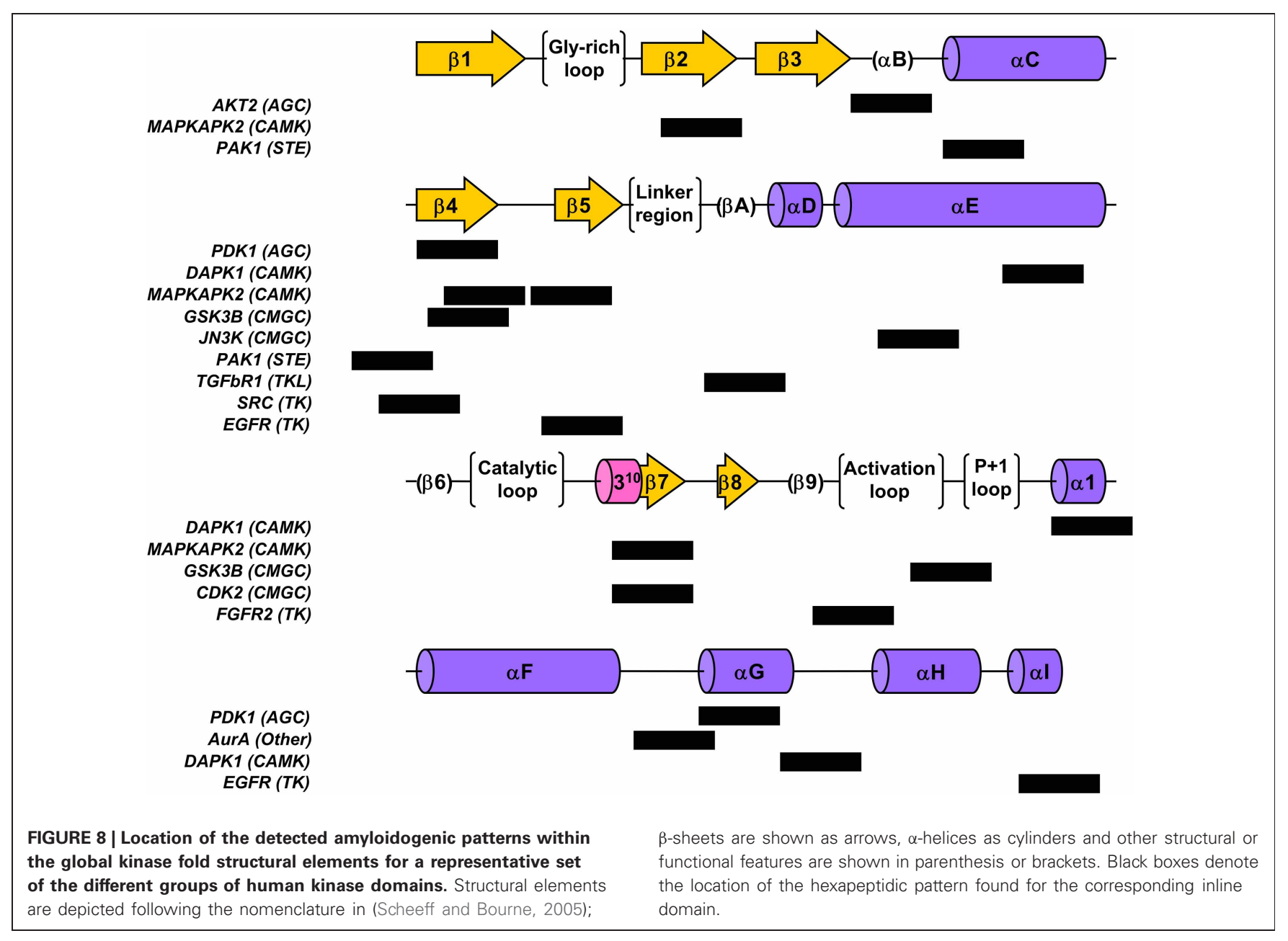

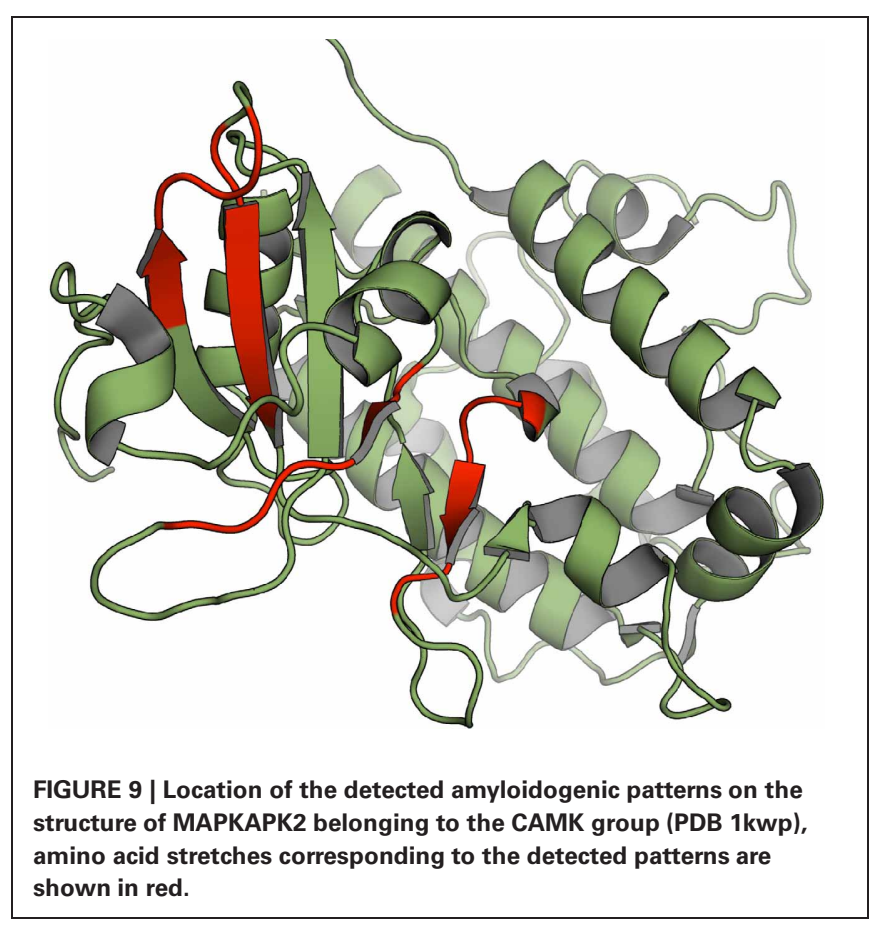

sequences and structures, the datasets used in these studies comprise proteins displaying divergent structures, from globular to unfolded, and functions, from essential to spurious, and thus under different evolutionary pressures.

Most evolutionary studies rely on the analysis of sequential or structural differences in a given protein family or super-family. By analogy, the study of the aggregation properties of a homogeneous, but relatively large, group of proteins sharing the same fold and function in different organisms might help to confirm the constraint aggregation exerts in protein evolution. The cellular role of the proteins in this set should be important enough to be the subject of a significant selection against their aggregation, in order to avoid a deleterious loss of function. The superfamily of protein kinases is perfectly suited for this purpose. We could confirm here that, on the average, the kinase domains of more complex and longer living organisms tend to have lower aggregation propensities and less potent aggregating peaks in their sequence.

Our predictions argue that, independently of the considered organism and group, kinase domains have significant aggregation tendency. Accordingly, human domains display higher aggregation propensity than the selected ensemble of folded proteins. Interestingly enough, it has been recently shown that $60 \%$ of 


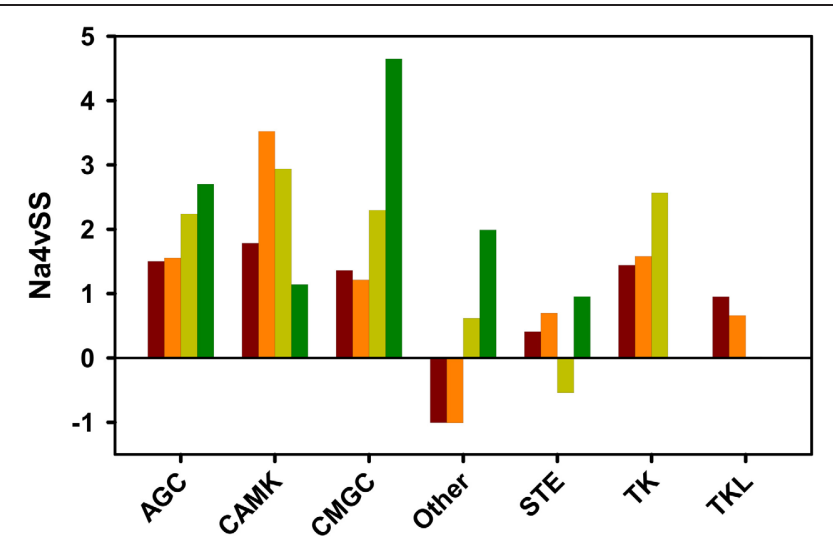

FIGURE 10 | Average aggregation propensity (Na4vSS) for discrete domain datasets of kinase groups in different organisms: human (brown), mouse (orange), fruit fly (light green), and budding yeast (dark green). Only the groups considered previously in the human kinome analysis are represented.

human kinases are clients of the HSP90 chaperone and that their binding determinants are located in the kinase domain (Taipale et al., 2012). In these cases, inhibition of the chaperone binding activity results in dissociation of the kinase domain and leads to aggregation, supporting the view that kinase domains are intrinsically aggregation-prone. This property might explain why, when recombinantly expressed in bacteria, kinase domains accumulate as misfolded and insoluble aggregates in many cases (Benetti et al., 1998; Marin et al., 2010) since prokaryotic HSP90 does not chaperone kinases (Buchner, 2010).

Despite the recognized cytoxicity of amyloid aggregates, it is now clear that amyloidogenic sequences are ubiquitous in all the proteomes (Castillo et al., 2011), supporting the view that most polypeptides share the potential to form amyloidassemblies (Dobson, 2004). Accordingly, we found that $85 \%$ of the human kinase domains include at least one amyloidogenic stretch and in many cases several of them. These data are in agreement with the observation that, in vitro, the population of partially unfolded states results in amyloid aggregation in different, unrelated, kinase domains (Damaschun et al., 2000; Agocs et al., 2010, 2012; Georgescauld et al., 2011). The mapping of these amyloidogenic regions on the three-dimensional structures of catalytic domains belonging to different human kinase groups indicates that, in most cases, they overlap with regular elements of secondary structure, providing support to the hypothesis that the molecular determinants responsible for amyloid formation coincide with those maintaining the native structure of proteins (Sabate et al., 2012). In other words, kinase domains cannot avoid the presence of amyloid regions simply because they need them to fold into compact and stable globular conformations. Moreover, aggregation-prone regions overlap with conserved functional residues, suggesting that the selection for active conformations during evolution constrains the pressure to attain more soluble sequences.

In the native state, the side-chains of amyloidogenic sequences are essentially protected from the solvent inside the three-dimensional structure of kinase domains. However, mutations or environmental conditions that destabilize the functional conformation would result in their partial or total exposition to solvent, allowing them to nucleate amyloid self-assembly, a mechanism common to several proteins involved in human conformational disorders (Monsellier and Chiti, 2007). This model would explain why certain kinases, such as c-Src and EGFR, associate transiently with HSP90 chaperone during maturation, where these regions are likely exposed to solvent, but do not bind to this chaperone when they are fully folded (Xu et al., 1999, 2005).

Despite the effect of selective pressure could be clearly traced for kinase domains, full-length kinases display, on the average, low aggregation propensity, independently of the considered species. Our analysis indicates that the presence of intrinsically unstructured regions in non-catalytic domains is, at least in part, responsible for the low sequential aggregation propensity of kinases. It has been recently shown that intrinsically disordered protein sequences traslationally fused to globular proteins act as entropic bristles, providing them solubility by creating both a large favorable surface area for water interactions and large excluded volumes around the partner (Santner et al., 2012). In kinases, this effect seems likely to act as a compensatory mechanism for the obligatory presence of aggregation-prone sequences in the catalytic domain.

Apart from structural and catalytic constrains, there is an alternative and, although speculative, more interesting explanation for the calculated aggregation propensity of kinase domains: that it serves for functional purposes. It has been shown that despite kinases can rapidly evolve away from chaperone assisted folding they have generally not done so. This suggests that kinases might specifically exploit association with the chaperone machinery as a means of regulation, in such a way that the inherent instability of some kinases might in fact be employed as a mechanism to provide fidelity in regulatory cascades (Taipale et al., 2012). The exposition of pre-existing aggregation-prone regions able to bind the chaperone upon partial unfolding would likely play an important role in this mechanism.

Despite the present work illustrates the utility of prediction algorithms to provide insights on the relationship between protein aggregation and sequence evolution, it should be noted that at the present moment these algorithms do not allow to evaluate the impact of post-translational modifications on aggregation propensity. It is clear that incorporating these modifications, which might promote extensive structural rearrangements, in the calculations would result in a more realistic readout on the evolutive constrains imposed by protein aggregation and that we should work toward this objective.

\section{MATERIALS AND METHODS DATASET CURATION}

Kinase Domains and Entire Protein sequences where retrieved from the Kinbase Database (http://kinase.com/kinbase/) for Homo sapiens, Mus musculus, Drosophila melanogaster, and Saccharomyces cerevisiae. Domains or proteins with "not available" ("N/A") sequence or containing the non-amino acidic 


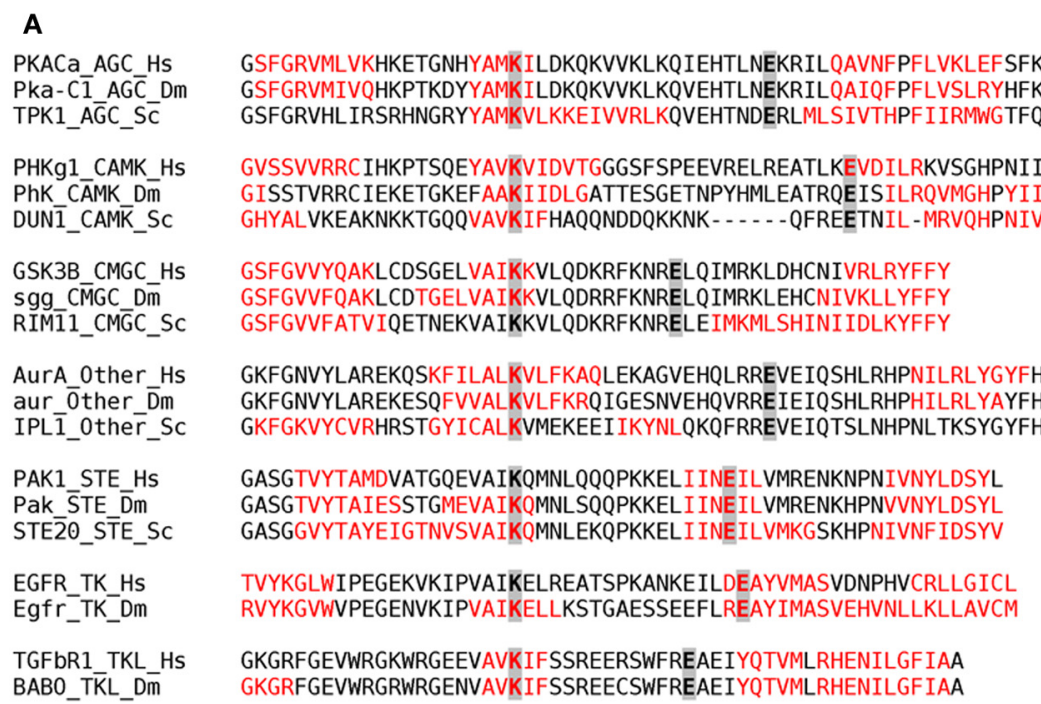

B

PKACa_AGC_Hs Pka- $\bar{C} \overline{1}$ AG $\bar{C}$ Dm TPK1_A $\bar{G} C \_S \bar{C}$

YAAOIVLTFEYLHSLDLIYRDLKPENLLIDQOGYIOVTDFGFAKRVKGRTWTLCGTPEY YAAQIVLAFEYLHYLDLIYRDLKPENLLIDSQGYLKVTDFGFAKRVKGRTWTLCGTPEY YAAEVCLALEYLHSKDI IYRDLKPENILLDKNGHIKITDFGFAKYVPDVTYTLCGTPDY

PHKgl_CAMK_Hs

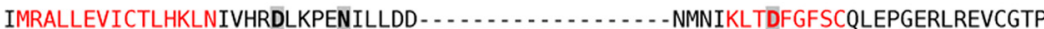

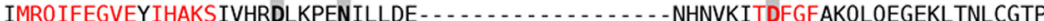
DUN1_CAMK_SC LFKQLLTGLKYLHEQNI IHRDIKPEN ILLNITRRENPSQVQLGPWDEDEIDIQVKIADFGLAKFTGEMQFTNTLCGTP

GSK3B CMGC Hs sgg CMGC Dm RIM1̄1_CMḠC_SC

YMYQLFRSLAYIHSF - GICHRDIKPQNLLLDPDTAVLKLCDFGSAKQLVRGEPNVSYICSR YMYOLFRSLAYIHSL - GICHRDIKPONLLLDPETAVLKLCDFGSAKOLLHGEPNVSYICSR YMFQLFKSLNYLHHFANVCHRDIKPQNLLVDPETWSLKLCDFGSAKQLKPTEPNVSYICSR

\section{AurA Other Hs} aur $\overline{0}$ ther $\bar{D} \mathrm{~m}$ IPLī_0ther $\bar{r}$ SC

YITELANALSYCHSKRVIHRDIKPENLLLGSAGELKIADFGWSVHA-PSSRRTTLCGTLD YIQALCSALLYLHERDI IHRDIKPENLLLGHKGVLKIADFGWSVHE-PNSMRMTLCGTVD YIYQIANALDYMHKKNI IHRDIKPEN ILIGFNNVIKLTDFGWSI INPPENRRKTVCGTID

PAK1 STE HS

Pak $\overline{\mathrm{S} T E} \overline{\mathrm{D}} \mathrm{m}$

STEZ̄o_STTE_SC

VCRECLQALEFLHSNQVIHRDIKSDNILLGMDGSVKLTDFGFCAQITPEQSKRSTMVGT VCREVLQALEFLHANQVIHRDIKSDN ILLGLDGSVKLTDFGFCAQISPEQSKRTTMVGT

TGFbR1_TKL_HS BABO_TKL_Dm VCRETLSGLEFLHSKGVLHRDIKSDNILLSMEGDIKLTDFGFCAOINELNLKRTTMVGT

EGFR TK Hs Egfr_TK_Dm

LAHLHMEIVGTQGKPAIAHRDLKSKNILVKKNGTCCIADLGLAVRHDSATDTIDIAPNH LAHLHMDIVGTRGKPAIAHRDLKSKNILVKSNLSCAIGDLGLAVRHVEKNDSVDIPSTH

WCVOIAKGMNYLEDRRLVHRDLAARNVLVKTPQHVKITDFGLAKLLGAEEKEYHAEGGK WSTQIAKGMSYLEEKRLVHRDLAARNVLVQTPSLVKITDFGLAKLLSSDSNEYKAAGGK

FIGURE 11 | Aggregation-prone regions detected by AGGRESCAN within the 20 amino acids flanking the Nter and Cter sides of the first $(A)$ and second $(B)$ regions containing conserved catalytic residues in kinase domains. Aggregation-prone stretches are shown in red in the alignment of representative proteins corresponding to different classes (AGC, CAMK, CMGC, Other, STE, TK, and TKL) in human (Hs), fruit fly $(\mathrm{Dm})$, and yeast (Sc). The conserved catalytic residues are highlighted in gray. character "*" were deleted. Whenever the non-amino acidic character "X" was found, it was substituted by alanine ("A"), unless more than three consecutive " $\mathrm{X}$ " were found, in which case the sequence was deleted. Single spaces found within sequences were suppressed.

Sequences in Kinase Domain datasets were verified to correspond to sequential regions of the Entire Protein datasets. Those domains without a correspondent sequence in the Entire Protein dataset were deleted. In the same way, full-length proteins without an associated kinase domain were also suppressed.

The Kinase Domain and Entire Protein datasets obtained in this way were further subdivided according to the different groups of kinases present in each species.
Due to AGGRESCAN technical limitations, parameters for sequences larger than 2000 amino acids could not be computed and those sequences and their corresponding domains were deleted from the datasets.

\section{AGGRESCAN CALCULATIONS}

AGGRESCAN (http://bioinf.uab.es/aggrescan/) was employed, with default settings, in order to compute parameters indicative of aggregation properties for every single sequence, as well as overall values for complete datasets. In order to be able to compare the large datasets of this study, only normalized values were considered (Figure 1). For further information about the calculation of AGGRESCAN parameters and its applications 
the reader is referred to AGGRESCAN Help File (accessible from the server front page) and to the published tutorials (de Groot et al., 2012).

\section{COMPOSITION OF KINASE DOMAINS}

The frequencies of occurrence of each amino acid were calculated for the complete kinase domains datasets of human and budding yeast. In order to look for compositional biases in these sets, frequency differences were calculated relative to the occurrence of each amino acid in the complete Swiss-Prot database release 2012_07.

\section{ANALYSIS OF THE HUMAN KINOME}

In order to be able to set up general comparisons between the human kinase proteins and its isolated domains, a reference dataset was defined by randomly retrieving, using a randomizing function, 500 sequences from the subset of sequences with less than $40 \%$ identity of the ASTRAL Compendium (Astral40), whose AGGRESCAN parameters were also calculated.

\section{REFERENCES}

Agocs, G., Solymosi, K., Varga, A., Modos, K., Kellermayer, M., Zavodszky, P., et al. (2010). Recovery of functional enzyme from amyloid fibrils. FEBS Lett. 584, 1139-1142.

Agocs, G., Szabo, B. T., Kohler, G., and Osvath, S. (2012). Comparing the folding and misfolding energy landscapes of phosphoglycerate kinase. Biophys. J. 102, 2828-2834.

Ball, C. A., Dolinski, K., Dwight, S. S., Harris, M. A., Issel-Tarver, L., Kasarskis, A., et al. (2000). Integrating functional genomic information into the Saccharomyces genome database. Nucleic Acids Res. 28, 77-80.

Belli, M., Ramazzotti, M., and Chiti, F. (2011). Prediction of amyloid aggregation in vivo. EMBO Rep. 12, 657-663.

Benetti, P. H., Kim, S. I., Chaillot, D., Canonge, M., Chardot, T., and Meunier, J. C. (1998). Expression and characterization of the recombinant catalytic subunit of casein kinase II from the yeast Yarrowia lipolytica in Escherichia coli. Protein Expr. Purif. 13, 283-290.

Buchner, J. (2010). Bacterial Hsp90desperately seeking clients. $\mathrm{Mol}$. Microbiol. 76, 540-544.

Castillo, V., Espargaro, A., Gordo, V., Vendrell, J., and Ventura, S. (2010). Deciphering the role of the thermodynamic and kinetic stabilities of SH3 domains on their aggregation inside bacteria. Proteomics 10, 4172-4185.

Castillo, V., Grana-Montes, R., Sabate, R., and Ventura, S. (2011).
Prediction of the aggregation propensity of proteins from the primary sequence: aggregation properties of proteomes. Biotechnol. J. 6, 674-685.

Castillo, V., and Ventura, S. (2009). Amyloidogenic regions and interaction surfaces overlap in globular proteins related to conformational diseases. PLoS Comput. Biol. 5:e1000476. doi: 10.1371/journal.pcbi.1000476

Chandonia, J. M., Hon, G., Walker, N. S., Lo Conte, L., Koehl, P., Levitt, M., et al. (2004). The ASTRAL Compendium in 2004. Nucleic Acids Res. 32, D189-D192.

Chen, Y., and Dokholyan, N. V. (2008). Natural selection against protein aggregation on self-interacting and essential proteins in yeast, fly, and worm. Mol. Biol. Evol. 25, 1530-1533.

Chiti, F., and Dobson, C. M. (2006). Protein misfolding, functional amyloid, and human disease. Annu. Rev. Biochem. 75, 333-366.

Conchillo-Sole, O., de Groot, N. S., Aviles, F. X., Vendrell, J., Daura, X., and Ventura, S. (2007). AGGRESCAN: a server for the prediction and evaluation of "hot spots" of aggregation in polypeptides. BMC Bioinformatics 8:65. doi: 10.1186/1471-2105-8-65

Daggett, V., and Fersht, A. R. (2009). Protein folding and binding: moving into unchartered territory. Curr. Opin. Struct. Biol. 19, $1-2$.

Damaschun, G., Damaschun, H., Fabian, H., Gast, K., Krober, R., Wieske, M., et al. (2000). Conversion of yeast

\section{DETECTION OF AMYLOIDOGENIC PATTERNS}

The human kinase domains dataset was explored to identify amyloidogenic patterns by using the amyloidogenic signature $\{\mathrm{P}\}$ $\{$ PKRHW $\}$-[VLSWFNQ]-[ILTYWFN]-[FIY]- $\{$ PKRH $\}$ defined in (de la Paz and Serrano, 2004) using the ScanProsite web server.

\section{INTRINSICAL UNFOLDING PREDICTION}

The intrinsical unfolding potential of selected subsets of the human and yeast kinase domains datasets was computed using the FoldIndex webserver with default settings.

\section{ACKNOWLEDGMENTS}

This work was supported by BFU2010-14901 from Ministerio de Ciencia e Innovacion (MCISpain) and 2009-SGR from AGAUR (Generalitat de Catalunya). Ricardo GrañaMontes is beneficiary of predoctoral FPU AP 20090948 fellowship from the Ministerio de Educación-Spain. Salvador Ventura has been granted an ICREA ACADEMIA award.

phosphoglycerate kinase into amyloid-like structure. Proteins 39, 204-211.

Dayhoff, M. O., Park, C. M., and McLaughlin, P. J. (1972). Atlas of Protein Sequence and Structure. Silver Spring, MD: National Biomedical Research Foundation.

de Groot, N., Pallares, I., Aviles, F., Vendrell, J., and Ventura, S. (2005). Prediction of "hot spots" of aggregation in disease-linked polypeptides. BMC Struct. Biol. 5:18. doi: 10.1186/1472-6807-5-18

de Groot, N. S., Castillo, V., GranaMontes, R., and Ventura, S. (2012). AGGRESCAN: method, application, and perspectives for drug design. Methods Mol. Biol. 819, 199-220.

de Groot, N. S., Sabate, R., and Ventura, S. (2009). Amyloids in bacterial inclusion bodies. Trends Biochem. Sci. 34, 408-416.

de Groot, N. S., and Ventura, S. (2010). Protein aggregation profile of the bacterial cytosol. PLoS ONE 5:e9383. doi: 10.1371/ journal.pone.0009383

de la Paz, M. L., and Serrano, L. (2004). Sequence determinants of amyloid fibril formation. Proc. Natl. Acad. Sci. U.S.A. 101, 87-92.

Dobson, C. M. (2003). Protein folding and misfolding. Nature 426, 884-890.

Dobson, C. M. (2004). Principles of protein folding, misfolding and aggregation. Semin. Cell Dev. Biol. 15, 3-16.

Georgescauld, F., Sabate, R., Espargaro, A., Ventura, S., Chaignepain, S., Lacombe, M. L., et al. (2011). Aggregation of the neuroblastoma-associated mutant (S120G) of the human nucleoside diphosphate kinase-A/NM23-H1 into amyloid fibrils. NaunynSchmiedeberg's Arch. Pharmacol. 384, 373-381.

Hamodrakas, S. J. (2011). Protein aggregation and amyloid fibril formation prediction software from primary sequence: towards controlling the formation of bacterial inclusion bodies. FEBS J. 278, 2428-2435.

Ivanova, M. I., Thompson, M. J., and Eisenberg, D. (2006). A systematic screen of beta(2)-microglobulin and insulin for amyloid-like segments. Proc. Natl. Acad. Sci. U.S.A. 103, 4079-4082.

Jahn, T. R., and Radford, S. E. (2005). The yin and yang of protein folding. FEBS J. 272, 5962-5970.

Linding, R., Schymkowitz, J., Rousseau, F., Diella, F., and Serrano, L. (2004). A comparative study of the relationship between protein structure and beta-aggregation in globular and intrinsically disordered proteins. J. Mol. Biol. 342, 345-353.

Manning, G., Plowman, G. D., Hunter, T., and Sudarsanam, S. (2002a). Evolution of protein kinase signaling from yeast to man. Trends Biochem. Sci. 27, 514-520.

Manning, G., Whyte, D. B., Martinez, R., Hunter, T., and Sudarsanam, S. (2002b). The protein kinase complement of the human genome. Science 298, 1912-1934.

Marin, V., Groveman, B. R., Qiao, H., Xu, J., Ali, M. K., Fang, X. Q., et al. (2010). Characterization of neuronal Src kinase purified from a 
bacterial expression system. Protein Expr. Purif. 74, 289-297.

Monsellier, E., and Chiti, F. (2007). Prevention of amyloid-like aggregation as a driving force of protein evolution. EMBO Rep. 8, 737-742.

Monsellier, E., Ramazzotti, M., Taddei, N., and Chiti, F. (2008). Aggregation propensity of the human proteome. PLoS Comput. Biol. 4:e1000199. doi: 10.1371/journal.pcbi.1000199

Nelson, R., and Eisenberg, D. (2006). Recent atomic models of amyloid fibril structure. Curr. Opin. Struct. Biol. 16, 260-265.

Rousseau, F., Schymkowitz, J., and Serrano, L. (2006a). Protein aggregation and amyloidosis: confusion of the kinds? Curr. Opin. Struct. Biol. $16,118-126$.

Rousseau, F., Serrano, L., and Schymkowitz, J. W. (2006b). How evolutionary pressure against protein aggregation shaped chaperone specificity. J. Mol. Biol. 355, 1037-1047.

Sabate, R., Espargaro, A., GranaMontes, R., Reverter, D., and Ventura, S. (2012). Native structure protects SUMO proteins from aggregation into amyloid fibrils. Biomacromolecules 13, 1916-1926.

Santner, A. A., Croy, C. H., Vasanwala, F. H., Uversky, V. N., Van, Y.
Y., and Dunker, A. K. (2012). Sweeping away protein aggregation with entropic bristles: intrinsically disordered protein fusions enhance soluble expression. Biochemistry 51, 7250-7262.

Scheeff, E. D., and Bourne, P. E. (2005). Structural evolution of the protein kinase-like superfamily. PLoS Comput. Biol. 1:e49. doi: 10.1371/journal.pcbi.0010049

Taipale, M., Krykbaeva, I., Koeva, M., Kayatekin, C., Westover, K. D., Karras, G. I., et al. (2012). Quantitative analysis of hsp90client interactions reveals principles of substrate recognition. Cell 150, 987-1001.

Tartaglia, G. G., and Caflisch, A. (2007). Computational analysis of the S. cerevisiae proteome reveals the function and cellular localization of the least and most amyloidogenic proteins. Proteins 68, 273-278.

Tartaglia, G. G., Pechmann, S., Dobson, C. M., and Vendruscolo, M. (2007). Life on the edge: a link between gene expression levels and aggregation rates of human proteins. Trends Biochem. Sci. 32, 204-206.

Tartaglia, G. G., Pellarin, R., Cavalli, A., and Caflisch, A. (2005). Organism complexity anti-correlates with proteomic beta-aggregation propensity. Protein Sci. 14, 2735-2740.
Tartaglia, G. G., and Vendruscolo, M. (2009). Correlation between mRNA expression levels and protein aggregation propensities in subcellular localisations. Mol. Biosyst. 5, 1873-1876.

Taylor, S. S., and Radzio-Andzelm, E. (1994). Three protein kinase structures define a common motif. Structure 2, 345-355.

Ventura, S., Zurdo, J., Narayanan, S., Parreno, M., Mangues, R., Reif, B., et al. (2004). Short amino acid stretches can mediate amyloid formation in globular proteins: the Src homology 3 (SH3) case. Proc. Natl. Acad. Sci. U.S.A. 101, 7258-7263.

Villar-Pique, A., de Groot, N. S., Sabate, R., Acebron, S. P., Celaya, G., Fernandez-Busquets, et al. (2012). The effect of amyloidogenic peptides on bacterial aging correlates with their intrinsic aggregation propensity. J. Mol. Biol. 421, 270-281.

Xu, W., Yuan, X., Xiang, Z., Mimnaugh, E., Marcu, M., and Neckers, L. (2005). Surface charge and hydrophobicity determine ErbB2 binding to the Hsp90 chaperone complex. Nat. Struct. Mol. Biol. 12, 120-126.

$\mathrm{Xu}$, Y., Singer, M. A., and Lindquist, $\mathrm{S}$. (1999). Maturation of the tyrosine kinase c-src as a kinase and as a substrate depends on the molecular chaperone Hsp90. Proc. Natl. Acad. Sci. U.S.A. 96, 109-114.

Conflict of Interest Statement: The authors declare that the research was conducted in the absence of any commercial or financial relationships that could be construed as a potential conflict of interest.

Received: 01 August 2012; accepted: 31 October 2012; published online: 20 November 2012.

Citation: Graña-Montes $R$, Sant'Anna de Oliveira $R$ and Ventura $S$ (2012) Protein aggregation profile of the human kinome. Front. Physio. 3:438. doi: 10.3389/fphys.2012.00438

This article was submitted to Frontiers in Systems Biology, a specialty of Frontiers in Physiology.

Copyright (c) 2012 Graña-Montes, Sant'Anna de Oliveira and Ventura. This is an open-access article distributed under the terms of the Creative Commons Attribution License, which permits use, distribution and reproduction in other forums, provided the original authors and source are credited and subject to any copyright notices concerning any third-party graphics etc.

\section{Research Topic:}

From structural to molecular systems biology: experimental and computational approaches to unravel mechanisms of kinase activity regulation in cancer and neurodegeneration
Topic Editor:

\section{Matteo Barberis,}

Humboldt University Berlin, Germany;

Max Planck Institute for Molecular Genetics, Berlin, Germany 